\title{
Mycobacterium senegalense Osteomyelitis of the Distal Tibia: A Case Report
}

\author{
Jeremiah Maupin ${ }^{1}$, Austin Cantrell1, Katherine Kupiec², Dante Paolo Melendez ${ }^{3}$, Amgad M. Haleem ${ }^{1,4}$ \\ 1. Department of Orthopedic Surgery and Rehabilitation, University of Oklahoma Health Sciences Center, Oklahoma City, Oklahoma, USA \\ 2. Department of Pharmacy, University of Oklahoma Medical Center, Oklahoma City, Oklahoma, USA \\ 3. Department of Internal Medicine, Infectious Disease Section, University of Oklahoma Health Sciences Center, Oklahoma City, Oklahoma, USA \\ 4. Department of Orthopedic Surgery, College of Medicine, Cairo University, Cairo, Egypt \\ $\square$ Corresponding author: Amgad M. Haleem MD, PhD, Amgad-Haleem@ouhsc.edu \\ (c) The authors. This is an open access article distributed under the terms of the Creative Commons Attribution (CC BY) license (https://creativecommons.org/licenses/ \\ by/4.0/). See http://ivyspring.com/terms for full terms and conditions.
}

Received: 2019.01.19; Accepted: 2019.05.01; Published: 2019.05.21

\begin{abstract}
Mycobacterium senegalense infection is rare. We present the third documented case of $M$. senegalense infection and the first to involve the musculoskeletal system. A 55-year old immunocompetent male developed chronic osteomyelitis of the ankle and required antibiotic spacers, an llizarov external fixator and multiple antibiotic regimens to eradicate the infection.
\end{abstract}

Key words: Mycobacterium senegalense; osteomyelitis; Nontuberculous mycobacterium; Ilizarov External Fixator

\section{Introduction}

Mycobacterium senegalense is a nontuberculous mycobacterium (NTM) species in the Mycobacterium fortuitum group best known for its devastating effects on cattle as one of the two leading causal agents of bovine farcy along with Mycobacterium farcinogenes.[1] Characterized by multiple abscesses, draining sinus tracts, and granuloma formation, bovine farcy leads to emaciation, death, and ultimately the inability to harvest beef from afflicted cattle causing both a disease and economic burden to ranchers.

While NTM infections occur in humans, it is difficult to predict an accurate incidence because unlike Mycobacterium tuberculosis, there is no mandatory health reporting for NTM infections.[2] NTM infections can affect multiple organ systems including the pulmonary, integumentary, hematologic and musculoskeletal systems.[3] More than 120 different species of NTM can cause human infection[2] and there is considerable difficulty in treating these infections. Many NTM species are resistant to routine anti-tuberculosis drugs, [4], [5] and with the slow growth rate of mycobacteria, patients often require prolonged durations of combination therapy which can lead to various side effects and difficulties tolerating treatment.

While human infection with $M$. senegalense appears to be rare with only two cases reported in the literature.[6],[7], there is likely an underestimation of the true incidence of these cases as some laboratories do not subspeciate $M$. fortuitum complex infections. The first reported case of $M$. senegalense occurred as a bacteremia from a central line infection in an immunocompromised 49 year-old female.[7] The second reported case occurred as a soft-tissue infection in a 3 year-old female who sustained facial lacerations from the glass of a freshwater fish tank.[6] Here, we present the third reported case of $M$. senegalense infection and the first to demonstrate musculoskeletal involvement in a 55 year-old male who developed chronic osteomyelitis following a traumatic open ankle fracture.

\section{Methods}

A literature search of the PubMed database was performed without time restrictions to locate reports of $M$. senegalense infection with the search term 
"senegalense." 65 total reports were identified spanning 1978-2018 and each was assessed for applicability to human infection. A total of 2 reports documented human infections and both were reviewed and referenced in this manuscript.
A

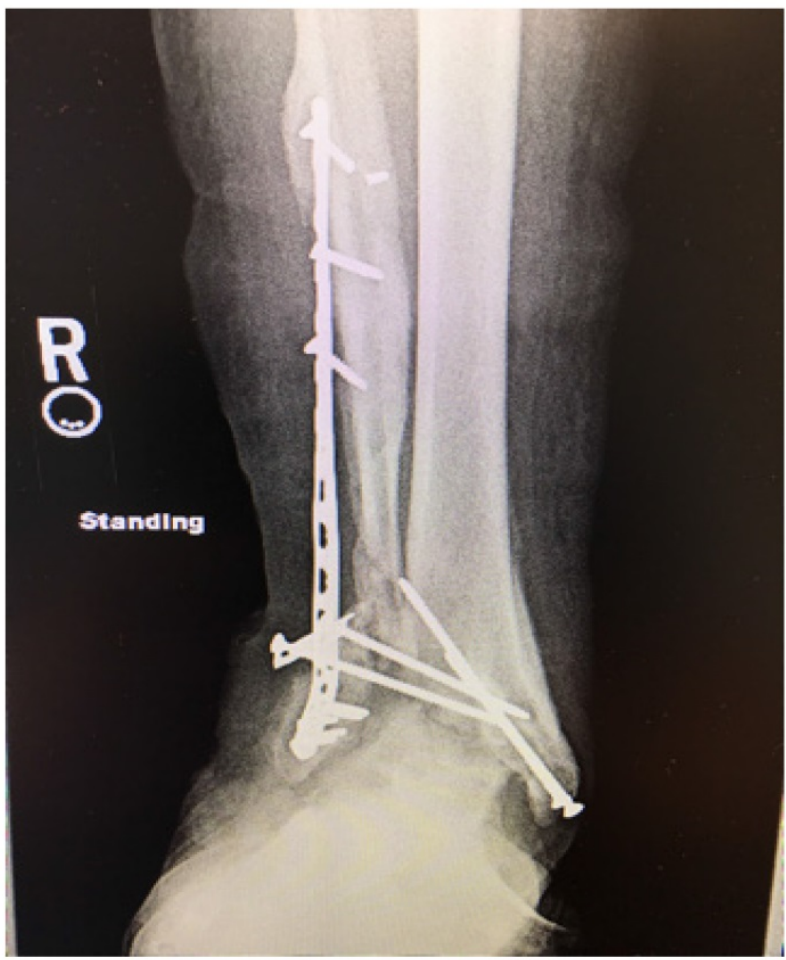

B

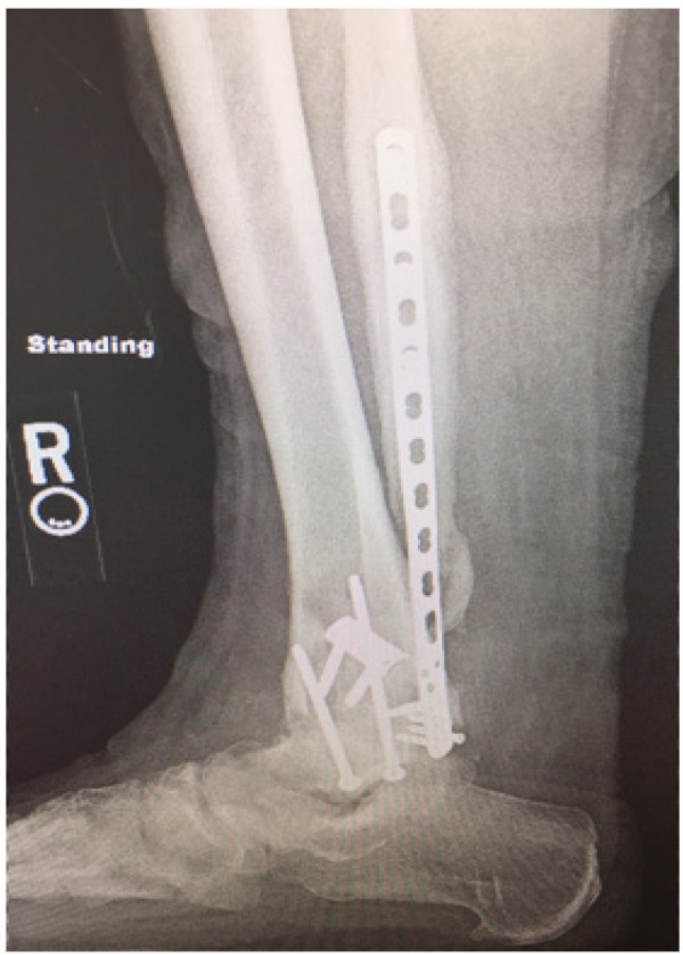

Figure 1: AP (A) and lateral (B) radiograph demonstrating hardware failure and destruction of the tibiotalar joint with severe valgus deformity and non-union

A

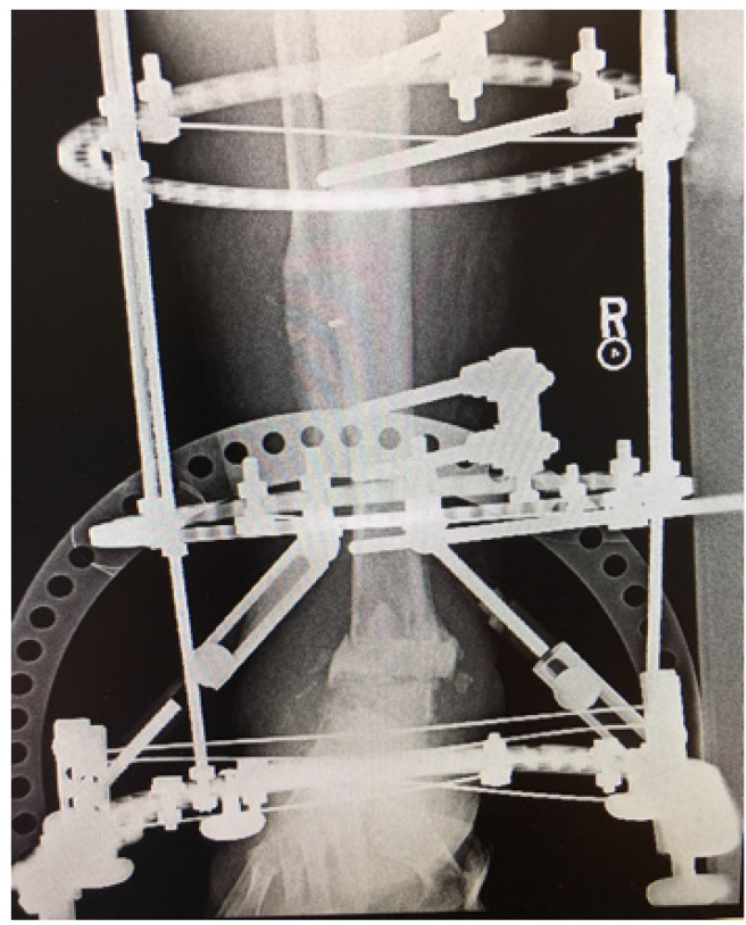

B

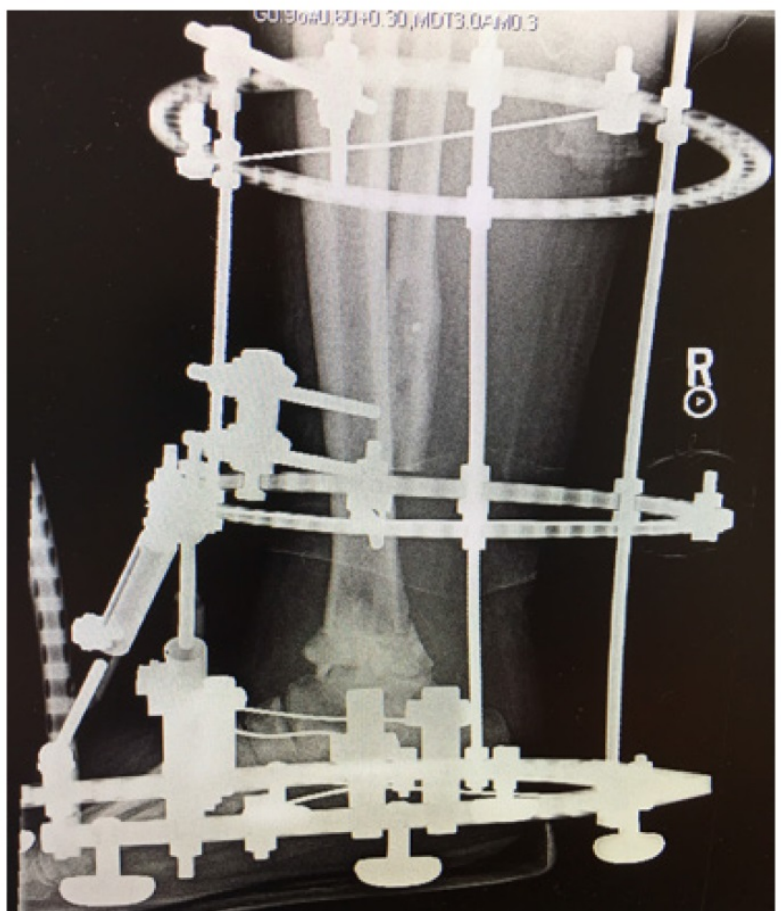

Figure 2: AP (A) and lateral (B) radiographs following removal of hardware, placement of tibiotalar antibiotic spacer and ringed external fixator 


\section{Case Report}

A 55-year-old immunocompetent male was involved in a motor vehicle collision 2 years prior to presenting to our institution. He sustained an open ankle fracture after being ejected from the driver's seat into a pasture ditch, sustaining lacerations from barbed wire and resulting in a grossly contaminated open fracture. He was initially managed with irrigation and debridement (I\&D) and placement of an external fixator. He subsequently underwent definitive fixation but during the next two years, experienced episodic drainage from incisions that was managed symptomatically with oral antibiotics. He had persistent pain which led him to seek evaluation at our institution.

Upon presentation to our clinic, he was noted to have a severe planovalgus alignment, two draining sinuses, radiographic hardware failure (Figure 1), and elevated inflammatory markers including a white blood cell (WBC) count of $11 \mathrm{~K} / \mathrm{mm}^{3}$, C-reactive protein (CRP) of $18.7 \mathrm{mg} / \mathrm{L}$, and Erythrocyte Sedimentation Rate (ESR) of $18 \mathrm{~mm} / \mathrm{hr}$. He was diagnosed with an infected nonunion with underlying chronic septic arthritis of the tibiotalar joint and osteomyelitis of the distal tibia and fibula and scheduled for a staged ankle fusion following eradication of his infection. At our index surgery, the patient underwent a complete hardware removal with the exception of a broken screw tip from the most proximal fibular screw, extensive debridement of the ankle including excision of 11 centimeters $(\mathrm{cm})$ of diseased distal fibula, $1.5 \mathrm{~cm}$ of tibial plafond, and $0.5 \mathrm{~cm}$ of talar dome, placement of an antibiotic spacer to the residual tibiotalar joint (impregnated with 2 grams of vancomycin and 2.4 grams of tobramycin per 40 grams of cement), and application of a ringed (Ilizarov) external fixator (Figure 2). Intraoperatively, multiple tissue cultures were obtained and found to be positive for Enterococcus faecalis, Enterobacter cloacae, and Mycobacterium senegalense, which grew from regular bacterial cultures.

Postoperatively, the patient was started on a 4-week regimen of piperacillin-tazobactam for the $E$. faecalis and E. cloacae osteomyelitis followed by a 6-week regimen of imipenem, ciprofloxacin, and minocycline for his $M$. senegalense based on the in vitro antibiotic susceptibilities from the culture (Table 1). Three months after his index surgery, he continued to have drainage from 2 sites and the decision was made to undergo a repeat I\&D with placement of a new antibiotic spacer (impregnated with vancomycin and tobramycin). Intraoperatively, the distal tibia had the appearance of persistent infection including purulence and caseous material which was cultured and noted to be positive for Bacillus species (not anthracis) and persistent $M$. senegalense growth, again from regular bacterial cultures (Table 1). At this point, based on the recurrent isolation of $M$. senegalense, the decision was made to continue imipenem and change the oral antibiotics to linezolid and azithromycin for another three months. Finally, after finishing 3 more months of imipenem, it was replaced with doxycycline to complete another 3 months of an all oral regimen.

Four months later, he had closure of all wounds, demonstrated downtrending inflammatory markers (WBC, ESR, CRP), and was felt to be ready for fusion. During the procedure, no purulence was encountered, iliac crest autograft was utilized to augment the tibiotalar fusion and compression through the joint was achieved through the ringed external fixator (Figure 3). Multiple tissue cultures were taken intraoperatively with no growth noted and postoperatively he was continued on his regimen of linezolid, azithromycin, and doxycycline.

Six months postoperatively, he had a successful fusion with no sinus tracts or drainage (Figure 4). He was able to have his ringed external fixator removed and one year postoperatively the patient continues to do well, fully weightbearing through the extremity and has not shown any signs of recurrence (Figure 5).

Table 1: Culture sensitivities from Mycobacterium senegalense isolates

\begin{tabular}{|c|c|c|c|c|}
\hline Antibiotic & $\begin{array}{l}\text { Index } \\
\text { Culture } \\
\text { MIC } \\
(\mathrm{mcg} / \mathrm{mL})\end{array}$ & $\begin{array}{l}\text { Interpretati } \\
\text { on }\end{array}$ & $\begin{array}{l}\text { Second } \\
\text { Culture } \\
\text { MIC } \\
(\mathrm{mcg} / \mathrm{mL})\end{array}$ & $\begin{array}{l}\text { Interpreta } \\
\text { tion }\end{array}$ \\
\hline Amikacin & $<=8$ & S & $<=8$ & S \\
\hline Amoxicillin-Clavulanate & $8 / 4$ & $\mathrm{~S}$ & $16 / 8$ & I \\
\hline Azithromycin & $<=16$ & S & $<=16$ & S \\
\hline Cefepime & $>32$ & $\mathrm{R}$ & $>32$ & $\mathrm{R}$ \\
\hline Cefotaxime & $>64$ & $\mathrm{R}$ & $>64$ & $\mathrm{R}$ \\
\hline Cefoxitin & 32 & I & 32 & $\mathrm{R}$ \\
\hline Ciprofloxacin & $<=1$ & S & $<=1$ & $S$ \\
\hline Clarithromycin & $<=0.25$ & S & $<=0.25$ & S \\
\hline Clofazimine & $<=0.5$ & $S$ & $<=0.5$ & $S$ \\
\hline Ceftriaxone & $>64$ & $\mathrm{R}$ & $>64$ & $\mathrm{R}$ \\
\hline Doxycycline & $<=1$ & $S$ & $<=1$ & S \\
\hline Gentamicin & $<=2$ & $S$ & $<=2$ & $S$ \\
\hline Imipenem & $<=2$ & $S$ & $<=2$ & $S$ \\
\hline Kanamycin & $<=8$ & $S$ & $<=8$ & $S$ \\
\hline Linezolid & 4 & S & 4 & $S$ \\
\hline Minocycline & $<=1$ & $S$ & $<=1$ & $S$ \\
\hline Moxifloxacin & $<=0.5$ & $S$ & $<=0.5$ & $S$ \\
\hline Tigecycline & $<=0.25$ & $S$ & 0.25 & $S$ \\
\hline Tobramycin & 4 & S & 4 & S \\
\hline $\begin{array}{l}\text { Trimethoprim-Sulfametho } \\
\text { xazole }\end{array}$ & $4 / 76$ & $\mathrm{R}$ & $1 / 19$ & S \\
\hline $\begin{array}{l}\text { *MIC-Minimum Inhibitory } \\
\text { I-Indeterminate }\end{array}$ & Concentra & ; S-Sensiti & R-Resista & \\
\hline
\end{tabular}




\section{Discussion}

Originally thought to be saprophytic bacteria, it was not until the 1950's that NTM species were recognized as human pathogens.[8] Now, there have been more than 120 different species of NTM identified that are capable of causing human disease.[2] The increased incidence of NTM infections is thought to be partially attributable to the genesis of the Acquired Immunodeficiency Syndrome (AIDS) epidemic, the use of immunosuppressive therapies, and the more widespread use of mycobacterial cultures and molecular identification methods in the laboratory.[9] The significant increase in the incidence of infections over the past 60 years, the associated disease burden that comes with management of these infections, and the increased resistance of NTM infections to routine antituberculosis drugs has led NTM species to gain acknowledgement as important pathogens of disease [4],[5].

This case demonstrates how difficult NTM infections can be to treat and the importance of a multidisciplinary team in management. From initial presentation to our facility, it was nearly a full year of both surgical and medical management before the infection was eradicated. While NTM species have the potential to be a contaminant, we maintain confidence that $M$. senegalense was causing infection in our case as it was isolated on two separate occasions 3 months apart.
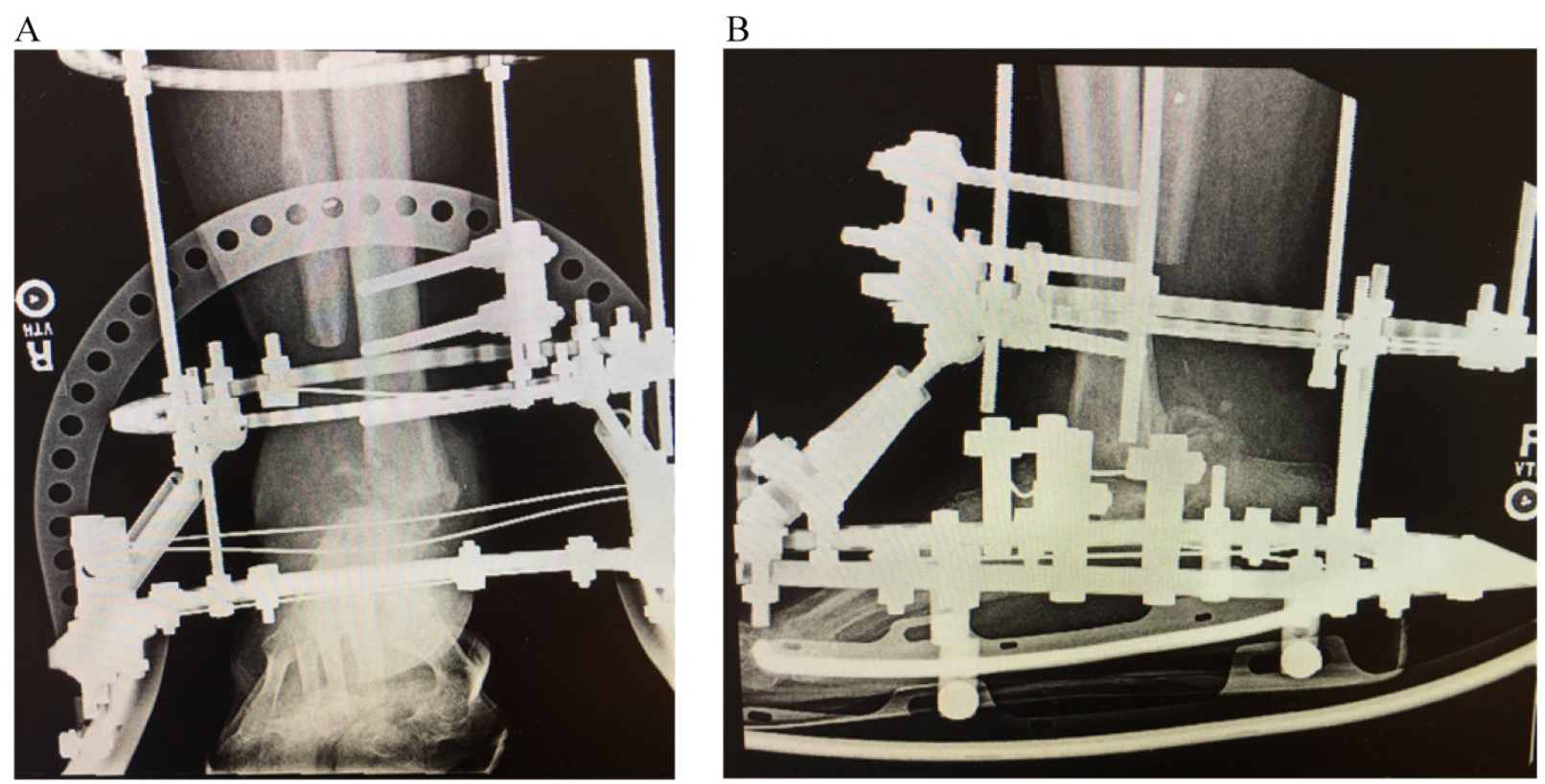

Figure 3: AP (A) and lateral (B) radiographs following definitive ankle fusion after eradication of infection

A

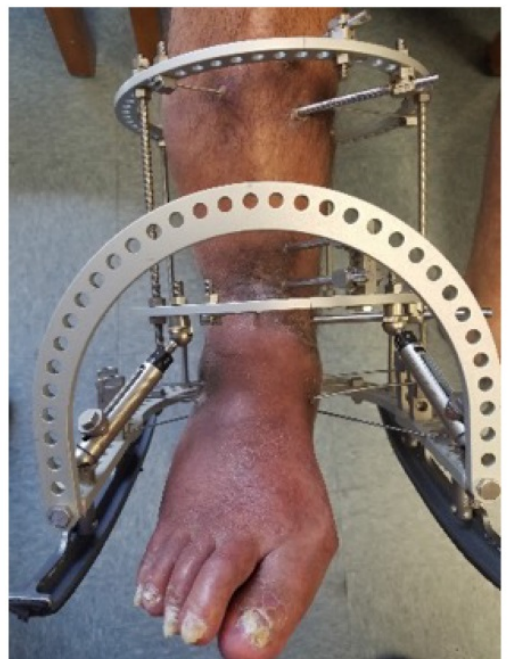

B

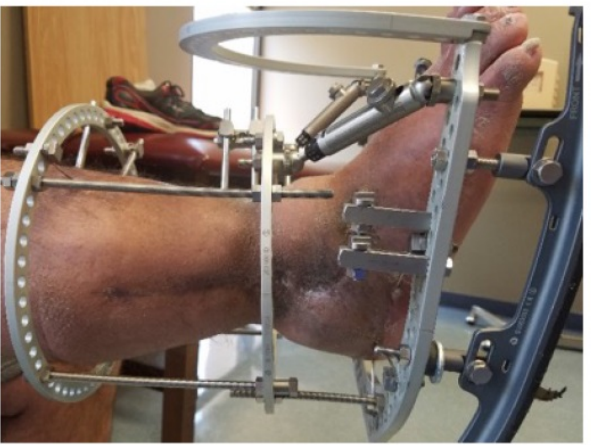

$\mathrm{C}$

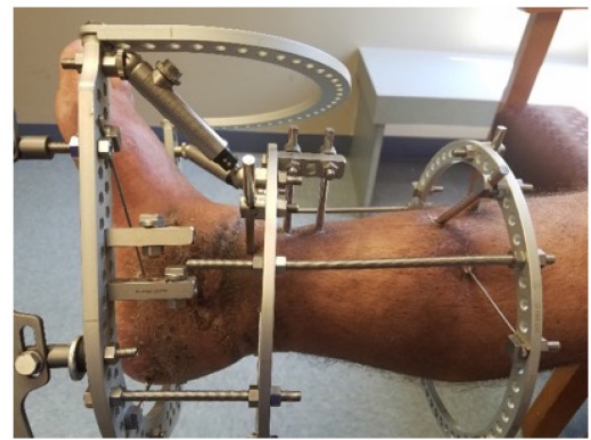

Figure 4: Right lower extremity (A-C) following successful infection eradication and tibiotalar fusion demonstrating healing of prior surgical sites and sinus tracts. 

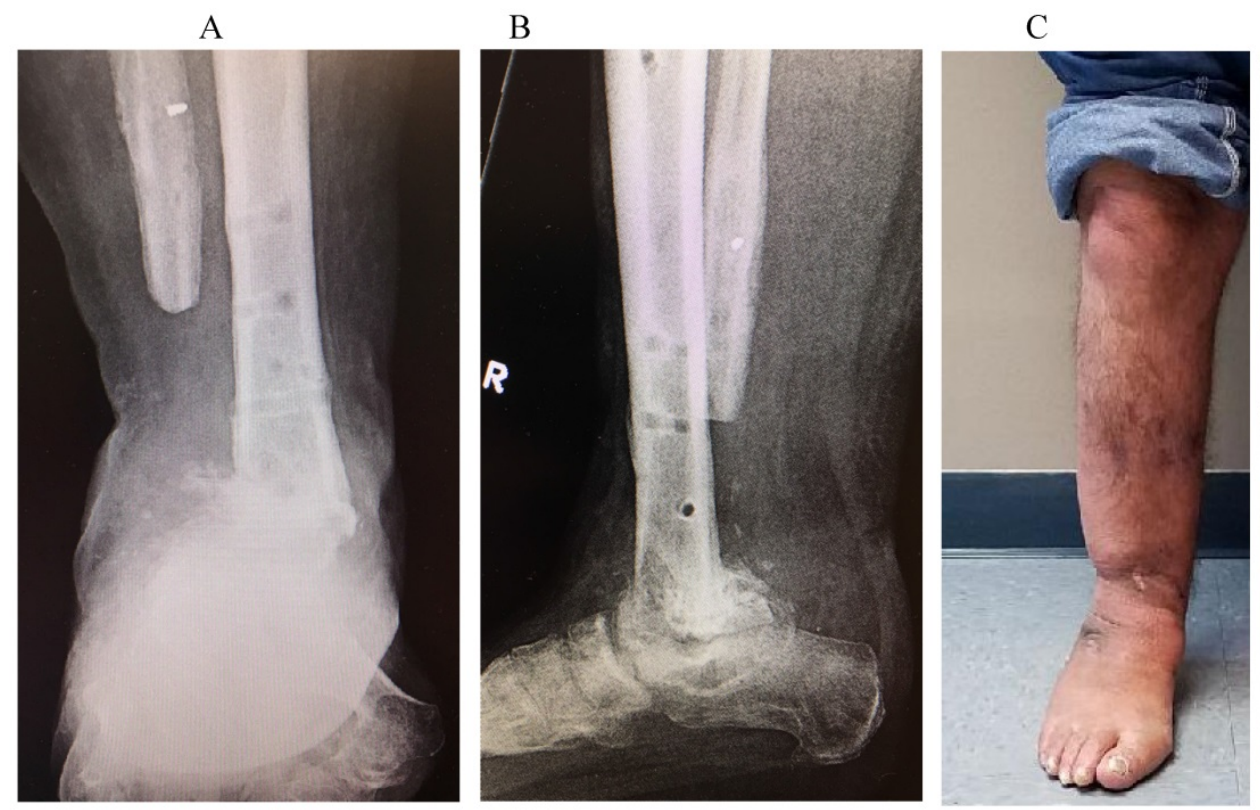

$\mathrm{D}$

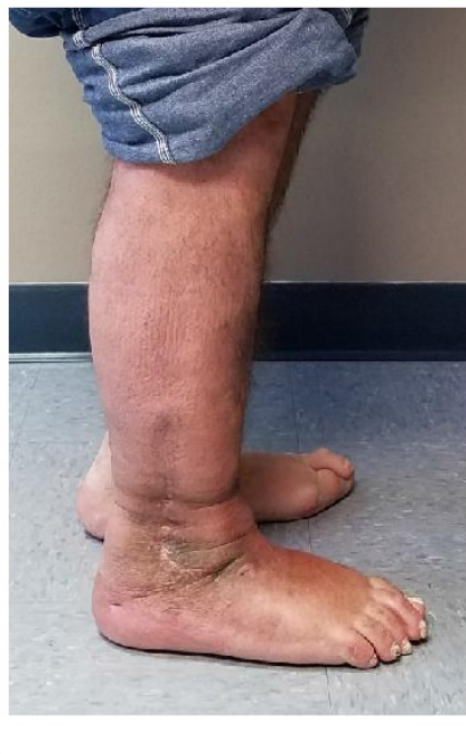

Figure 5: AP (A) and Lateral (B) radiographs with same day clinical appearance of extremity (C \& $D)$ 1-year following fusion demonstrating a solid tibiotalar fusion mass and well healed incisions with no evidence of infection recurrence.

As literature on M. senegalense infection is sparse and limited to two prior case reports, we based our antimicrobial management on the available guidelines for NTM infection treatment on $M$. fortuitum group, to which $M$. senegalense belongs.[10] Thus, we treated with a combination of a minimum of 2 antimicrobials specifically directed to this isolate of $M$. senegalense, guided by in vitro susceptibilities.

While macrolides are the backbone in the treatment of most NTM infections, current treatment guidelines by the American Thoracic Society/Infectious Diseases Society of America recommend caution in their use when treating infections by the $M$. fortuitum group, given the potential for development of resistance through an inducible erythromycin methylase erm gene.[10] Testing for this erm gene-related resistance requires incubation of the mycobacterial isolate in the presence of a macrolide for 14 days before the assessment of its minimum inhibitory concentration. This technique is unfortunately not widely available and as such, was not available in this case. While the initial antibiotic regimen consisted of 3 non-macrolide agents, the definitive antibiotic regimen included a macrolide in addition to other 2 antibiotics, one of which was administered intravenously. We cautiously used the macrolide antibiotic only as an addition to the other 2 non-macrolide antibiotics and after extensive surgical debridement had been performed. This multimodal approach of thorough surgical debridement with removal of infected hardware and combination antibiotic therapy is key in the treatment of NTM infections and in our opinion decreased substantially the risk for development of macrolide resistance and increased the chances of success. To further illustrate this point, the previously reported $M$ senegalense catheter-related infection failed initial conservative management but was successfully treated only after fully removing the infected hardware [7].

From a surgical standpoint, chronic osteomyelitis of long bones can be a serious and frustrating problem to treat as there is no generally accepted consensus on management. At our institution, we typically manage cases of chronic osteomyelitis in a staged fashion, allowing us to confirm eradication prior to definitive surgery. For cases like this with significant periarticular joint destruction, we favor the use of an Ilizarov external fixator which allows us the versatility to maintain the hardware away from the infected tissues while simultaneously allowing us access to the site of infection for serial debridements, such as in this case where an antibiotic spacer exchange was necessary. Furthermore, the Ilizarov external fixator has the added benefit of being capable of generating compression across the fusion sites at the time of definitive surgery, as well as post-operatively in an outpatient setting if needed. Prior studies have shown success managing chronic osteomyelitis with Ilizarov frames when combined with appropriate antibiotic therapy. While there has not been literature specifically addressing NTM infections managed with Ilizarov external fixators, eradication was achieved in our case and we anticipate success rates would be similar to those of prior published studies [11],[12]. 


\section{Conclusion}

Initially thought to be benign saprophytes, nontuberculous mycobacterium species are now a well-known source of human infection. This case demonstrates the third documented case of human Mycobacterium senegalense infection and is the first to be associated with osteomyelitis. Through a multidisciplinary approach including infectious disease specialists and orthopedic surgeons, we were ultimately able to eradicate the infection and preserve the patient's extremity. We present this case to further add to the sparse body of literature on this species and create awareness that $M$. senegalense can be a source of osteomyelitis, even in an immunocompetent host.

\section{Author contributions}

$\mathrm{AMH}$ and DPM are the attending physicians for the patient and collected medical data for the patient. $\mathrm{JM}$ is the resident physician for the patient. KK is the pharmacist consulting on the case. JM, AC, KK, DPM, and $\mathrm{AMH}$ all contributed to the manuscript. All authors have read and approved the final manuscript.

\section{Abbreviations}

NTM: nontuberculous mycobacterium; I\&D: irrigation and debridement; WBC: white blood cell; CRP: C-reactive protein; ESR: Erythrocyte Sedimentation Rate; AIDS: Acquired Immunodeficiency Syndrome; MIC: minimum inhibitory concentration.

\section{Competing Interests}

The authors have declared that no competing interest exists.

\section{References}

1. Hamid ME. Current Perspectives on Mycobacterium farcinogenes and Mycobacterium senegalense, the Causal Agents of Bovine Farcy. Veterinary medicine international. 2014; 2014: 247906.

2. Hogan JI, Hurtado RM, Nelson SB. Mycobacterial Musculoskeletal Infections. Infectious disease clinics of North America. 2017; 31: 369-82.

3. Petitjean G, Fluckiger U, Scharen S, Laifer G. Vertebral osteomyelitis caused by non-tuberculous mycobacteria. Clinical microbiology and infection : the official publication of the European Society of Clinical Microbiology and Infectious Diseases. 2004; 10: 951-3.

4. Wallace RJ, Jr., O'Brien R, Glassroth J, Raleigh J, Dutt A. Diagnosis and Treatment of Disease Caused by Nontuberculous Mycobacteria. American Review of Respiratory Disease. 1990; 142: 940-53.

5. Gundavda MK, Patil HG, Agashe VM, Soman R, Rodriques C, Deshpande RB. Nontuberculous mycobacterial infection of the musculoskeletal system in immunocompetent hosts. Indian journal of orthopaedics. 2017; 51: 205-12.

6. Talavlikar R, Carson J, Meatherill B, Desai S, Sharma M, Shandro C, et al. Mycobacterium senegalense tissue infection in a child after fish tank exposure. The Canadian journal of infectious diseases \& medical microbiology = Journal canadien des maladies infectieuses et de la microbiologie medicale. 2011; 22: $101-3$.

7. Oh WS, Ko KS, Song JH, Lee MY, Ryu SY, Taek S, et al. Catheter-associated bacteremia by Mycobacterium senegalense in Korea. BMC infectious diseases. 2005; 5: 107

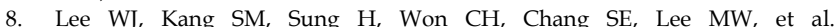
Non-tuberculous mycobacterial infections of the skin: a retrospective study of 29 cases. The Journal of dermatology. 2010; 37: 965-72.

9. Wagner D, Young LS. Nontuberculous mycobacterial infections: a clinical review. Infection. 2004; 32: 257-70.
10. Griffith DE, Aksamit T, Brown-Elliott BA, Catanzaro A, Daley C, Gordin F, et al. An official ATS/IDSA statement: diagnosis, treatment, and prevention of nontuberculous mycobacterial diseases. American journal of respiratory and critical care medicine. 2007; 175: 367-416.

11. Megas P, Saridis A, Kouzelis A, Kallivokas A, Mylonas S, Tyllianakis M. The treatment of infected nonunion of the tibia following intramedullary nailing by the Ilizarov method. Injury. 2010; 41: 294-9.

12. Yin P, Ji Q, Li T, Li J, Li Z, Liu J, et al. A Systematic Review and Meta-Analysis of Ilizarov Methods in the Treatment of Infected Nonunion of Tibia and Femur. PloS one. 2015; 10: e0141973. 